\title{
Income trajectories affect treatment of dental caries from childhood to young adulthood: a birth cohort study
}

\section{Marco Aurelio PERES(a) Pingzhou LIU(a) \\ Flavio Fernando DEMARCO ${ }^{(b, c)}$ \\ Alexandre Emidio Ribeiro SILVA(c) \\ Fernando Cesar WEHRMEISTER ${ }^{(\mathrm{c})}$ \\ Ana Maria MENEZES(c) \\ Karen Glazer PERES(a)}

(a)The University of Adelaide, Adelaide Dental School, Australian Research Centre for Population Oral Health, Adelaide, Australia.

(b) Universidade Federal de Pelotas - UFPel, Postgraduate Program in Dentistry, Pelotas, RS, Brazil.

(c) Universidade Federal de Pelotas - UFPel, Postgraduate Program in Epidemiology, Pelotas, RS, Brazil.

Declaration of Interests: The authors certify that they have no commercial or associative interest that represents a conflict of interest in connection with the manuscript.

Corresponding Author:

Marco A. Peres

E-mail:marco.peres@adelaide.edu.au

hitps://doi.org/10.1590/1807-3107bor-2018.vol32.0036

Submitted: December 12, 2017

Accepted for publication: February 27, 2018

Last revision: March 14, 2018

\begin{abstract}
We aimed to analyze the effects of family income trajectories on the increase in dental caries from childhood to young adulthood. Data from the 1993 Pelotas (Brazil) birth cohort study, in which dental caries was measured at ages 6,12 , and 18 years, were analyzed. Family income of 302 participants was assessed at birth, and at 4, 11, 15, and 18 years of age. Mother's education, toothbrushing frequency, dental visiting, dental caries in primary dentition, and birth weight were covariates. A latent class growth analysis was conducted to characterize trajectories of timevarying variables. The influence of income trajectories on the increase in dental caries from age 6 to age 18 was evaluated by a generalized linear mixed model. After adjustment, the increases in numbers of decayed and missing teeth (DMT) from age 6 to age 18 were associated with family income trajectory. The incident rate ratios (IRR) of DMT compared with the group of stable high incomes were 2.36 for stable low incomes, 1.71 for downward, and 1.64 for upward. The IRR of teeth being filled in stable lowincome groups compared with stable high-income groups was 0.55 . Family income mobility affected treatment patterns of dental caries. Differences across income trajectory groups were found in the components of dental caries indices rather than in the experience of disease.
\end{abstract}

Keywords: Dental Caries; Cohort Studies; Healthcare Disparities.

\section{Introduction}

Dental caries is a chronic, highly prevalent, and cumulative noncommunicable disease which develops over time. ${ }^{1,2}$ Untreated caries in permanent teeth was the most prevalent condition among those evaluated for the Global Burden of Disease 2010 Study. ${ }^{3}$ Moreover, social inequalities in oral health remain. A marked social gradient exists, and it is similar to that in general health. ${ }^{4}$ However, since most of the research on dental caries and socioeconomics is cross-sectional, it is not possible to capture how the dynamic of socioeconomic conditions in the life-course may influence the increase in dental caries throughout a lifetime.

Few prospective cohort studies have investigated dental caries and socioeconomic trajectories. . $^{1,5,6,7}$ All have analyzed cross-sectional dental caries and/or socioeconomic conditions. It is noticeable that very few studies in the health literature modeled socioeconomic trajectories ${ }^{8}$ and their effects on health outcomes. 
The role of socioeconomic mobility in the lifecourse on dental caries trajectories from childhood to young adulthood in the context of middle- or low-income countries is unknown. To fill this gap in the literature, we aimed to answer the following research question: Do family income trajectories in the life-course affect increases in dental caries from childhood to adulthood?

\section{Methodology}

This study is reported according to Strengthening the Reporting of ObservationalStudies in Epidemiology (STROBE) guidelines.

\section{Study population}

This study was nested in the 1993 Pelotas, Brazil, population-based birth cohort study. All hospitals in Pelotas were visited daily from 1 January to 31 December 1993. Detailed information regarding cohort and oral health methodological aspects has been published elsewhere. ${ }^{910,11}$ Briefly, all mothers who lived in urban areas and had given birth at these institutions were invited to take part in the study. The children included in the cohort $(n=5,249)$ represented 99\% of the total births in Pelotas. All mothers were asked about their social and economic conditions, demographics, pregnancy characteristics, healthrelated behaviors, health care, and morbidity, and the children were weighed, measured, and examined at birth. A random sub-sample of the cohort children was selected and visited in the subsequent follow-up waves, at the ages of $1(n=649), 3(n=644), 6(n=1,414)$, and $12(n=1,383)$ months. In 1997, a sample of the original cohort, including all low-birth-weight children, and a random $20 \%$ of the remainder were revisited. Of 1,460 eligible children, $87 \%$ (1270 children) were located.

\section{Oral health study at age $\mathbf{6}$ years}

A sub-sample, drawn from the 1998 follow-up group, was examined in 1999 for estimation of the occurrence of dental caries, anterior open bite, and posterior crossbite at age 6 , and also as a test of some hypotheses related to dental caries ${ }^{12}$ and malocclusion, ${ }^{13}$ resulting in a sample of 400 children. The oral health study included a dental examination with regard to dental caries in primary and permanent dentition, ${ }^{14}$ malocclusion, and oral mucosal lesions in addition to the administration of a questionnaire to the participants' mothers at home. The questionnaire sought information related to dental hygiene habits and the types and uses of dental services. Pre-tests of the questionnaire, examiner calibration of three dentists, and a pilot study were carried out before the field work among 40 children. Inter-examiner reliability was measured by the kappa statistic, showing 0.65 as the lowest value for the tooth-by-tooth diagnosis of the dental status, while the majority of the values equaled 1.0. The participation rate was $89.7 \%(n=359)$.

\section{Oral health study at age 12 years}

All of the 359 children who participated in the study at 6 years of age were visited in their homes in 2005, when they were 12 years old, and of those, 339 (94.4\%) were investigated. A questionnaire on the use of dental services, history of toothache, and habits and behaviors related to oral hygiene was administered to the adolescents. Four teams of fieldworkers were formed, each consisting of an examiner and an interviewer. Dental caries in permanent teeth was assessed according to the WHO diagnostic criteria. ${ }^{14}$ Examiner calibration was performed on a tooth-bytooth basis among 40 children aged 11-13 who did not take part in the cohort and followed the methodology previously described. ${ }^{15}$ Examiner reliability was assessed by means of weighted kappa for categorical variables in the same 40 children. The first author (MAP) was the gold standard for the 6- and 12-yearold dental caries examinations. The lowest value of 0.6 was considered substantial or good. ${ }^{16}$

\section{Oral health study at age 18 years}

When those dentally examined and interviewed at age 12 reached 18 years of age, we investigated 307 $(90.6 \%)$ of them. Only one trained dentist examined all participants and had high kappa values for interand intra-examiner agreement (weighted kappa 0.78 and 0.83 , respectively). Dental caries was assessed according to the WHO criteria, ${ }^{14}$ and a questionnaire seeking information on habits and patterns of dental visits was also administered. All examinations and interviews were conducted at the cohort clinic. 


\section{Outcomes}

The main outcome variable was dental caries experience in permanent teeth (DMFT) and was analyzed separately for decayed and missed teeth (DMT) and filled teeth (FT) at ages 6, 12, and 18 years. Dental caries in the primary dentition was assessed at age 6 years by $\mathrm{dmft}$. Caries experience indices were calculated by counting the numbers of $\mathrm{D}, \mathrm{M}$, and $\mathrm{F}$ teeth.

\section{Main explanatory variables}

Explanatory variables were gathered from the different cohort wave datasets. Family income of participants with dental data in the three waves (ages 6,12 , and 18 years) was assessed at birth and at 4,11 , 15 , and 18 years of age. Household income or family income is a measure that combines earnings in the month previous to the interview of all people sharing a household or place of residence. It includes every form of income, such as salaries, wages, rental income, retirement income, government cash transfers, and investment gains.

\section{Covariates}

Mother's education level was measured by the number of years at school recorded at three time points (participants' birth-year and ages 15 and 18). Daily toothbrushing frequency was collected at four time points (ages 6, 12,15, and 18 years). Dental visit was assessed at ages 6,12 , and 15. Birth weight was collected in grams and was considered as a proxy of children's general health. ${ }^{7}$

\section{Statistical analyses}

Caries experience was the main dependent variable, and its descriptive statistics were presented with caries prevalence (cases and proportions) and caries experience (means and standard errors). Family income was categorized into tertiles for each of five data-collecting years. Mother's education levels were categorized ( $0-4$, $5-8,9-11$, and $>12)$ according to completed school years at three time periods. Two categories $(<2$ per day and $\geq 2$ per day) of daily toothbrushing frequency were used for every time period. As in the follow-up study, the proportion of low-birth-weight children was $29.7 \%$; since, in the original cohort, it was $9.7 \%$, it was necessary to calculate a weighted factor to perform statistical analysis. Therefore, for the Oral Health Study a weight factor of 0.34 was used for children born with low birth weight, and 1.27 was applied for those born with adequate birth weight. Household incomes and other covariates were all described in cross-tables with their regrouped categories.

Group-based trajectory analysis was performed with PROC TRAJ macro in SAS version $9.3^{17}$ to characterize trajectories of time-varying independent variables that had at least three time points. The parameters for the trajectory model were determined on a maximum-likelihood basis by a general quasiNewton method. ${ }^{18,19}$ A four-group trajectory analysis model was defined for family incomes-three groups for mother's education and two groups for toothbrushing frequency. Selection of that number balanced the interests of parsimony with the objective of reporting the distinctive developmental patterns in the data. ${ }^{2}$ There was no clear trajectory developed from three waves of dental visits.

The model selection procedure involved estimation of the number of latent classes and the order of the polynomial for each latent trajectory. The final number of trajectories was established when sequential comparisons of the Bayesian Information Criterion (BIC) and adjusted BIC between the models with $\mathrm{k}$ and $k+1$ trajectories yielded no further substantial reductions in the BIC score than the $\mathrm{k}+1$ model. We fitted each group with the trajectory of quadratics and started with the null model with one group. BIC analysis supports a five-group model, but we chose the four-group model, due to two groups in the five-group model being hardly distinguishable from members' probabilities and features of the data. To determine the validity of the model, we searched for all possible models within the class for groups equal to four. From the maximum likelihood estimates (ML), the best-fit model was the one with the smallest BIC. Ultimately, a four-group income trajectory was selected with the orders $(1,2,2$, and 2) as for stable low-income (52.4\%), downward (8.9\%), upward (19.0\%), and stable high-income (19.7\%) (Figure 1b). The likelihood of each case belonging to each trajectory (posterior probabilities) was used to classify individual group membership. 
To identify the associated factors with the main exploratory variable-income trajectory groups -we fit GLMM with SAS PROC GLIMMIX by assuming negative binomial distribution with log link function (NB model) on three-times-repeated dental caries. The primary assumption underlying the analyses performed by PROC GLIMMIX on the data was that it contained random effects in dealing with repeated measures on each individual. The choice of the NB model is due to the positive skewness of the DMFT index. ${ }^{20}$ We used Type 3 likelihood statistics for testing the overall effects of the variables, and Wald Chi-square statistics tested the effect of category of variable compared with the reference group in the model. All variables with Type 3 likelihood statistics $p<0.20$ were kept in the models. Gender was kept in the models regardless of its $p$ value. The model allowed for the estimation of dental caries incidence rate ratio and respective $95 \%$ confidence intervals.
Least-squares means were estimated through the models. The estimated expected counts of dental caries were obtained by application of the inverse link function to the least-squares means.

\section{Ethical issues}

Consent for interviews and examinations was obtained and approved by the Pelotas Federal University Ethics Committee. Participants who presented with dental treatment needs were referred to the Dental Clinic of the Postgraduate Program in Dentistry of Pelotas Federal University.

\section{Results}

Of the 359 children investigated in the baseline, $302(83.8 \%)$ had completed data on dental caries, family income, and covariates at age 18 years. Table 1 displays the prevalence and experience of dental caries

Table 1. Sample characteristics studied in each wave.*

\begin{tabular}{|c|c|c|c|c|c|c|c|}
\hline Variable & Age 0 & Age 4 & Age 6 & Age 11 & Age 12 & Age 15 & Age 18 \\
\hline \multicolumn{8}{|l|}{ Caries prevalence n (\%) } \\
\hline $\mathrm{dmft}>0$ & - & - & $195(64.4)$ & - & - & - & - \\
\hline $\mathrm{DMT}>0$ & - & - & $9(2.8)$ & & $118(38.8)$ & & $148(48.8)$ \\
\hline $\mathrm{FT}>0$ & - & - & $2(0.4)$ & & $57(18.6)$ & & $134(44.4)$ \\
\hline DMFT $>0$ & - & - & $11(3.2)$ & & $153(50.3)$ & & $214(70.8)$ \\
\hline \multicolumn{8}{|l|}{ Caries experience mean (S.D.) } \\
\hline $\mathrm{dmft}$ & - & - & $3.46(3.9)$ & - & - & - & - \\
\hline DMT & - & - & $0.05(0.4)$ & - & $0.87(1.4)$ & - & $1.12(1.6)$ \\
\hline $\mathrm{FT}$ & - & - & $0.01(0.2)$ & - & $0.37(0.9)$ & - & $0.98(1.5)$ \\
\hline DMFT & - & - & $0.06(0.4)$ & - & $1.24(1.6)$ & - & $2.10(2.3)$ \\
\hline \multicolumn{8}{|l|}{ Family income (tertile) ${ }^{* *} \mathrm{n}(\%)$} \\
\hline Lower & $133(44.0)$ & $88(29.4)$ & - & 85 (28.3) & - & $80(26.6)$ & $80(26.7)$ \\
\hline Intermediate & $61(20.2)$ & $88(29.1)$ & - & $99(32.8)$ & - & $110(36.6)$ & $100(33.3)$ \\
\hline Higher & $106(35.3)$ & $125(41.5)$ & - & $117(38.9)$ & - & $111(36.9)$ & $121(40.1)$ \\
\hline \multicolumn{8}{|l|}{ Mother's schooling (Y) n (\%) } \\
\hline $0-4$ & $74(24.6)$ & - & - & - & - & $77(25.6)$ & 57 (18.9) \\
\hline $5-8$ & $145(48.1)$ & - & - & - & - & $129(42.8)$ & $129(42.8)$ \\
\hline $9-11$ & $60(19.9)$ & - & - & - & - & $85(28.3)$ & $85(28.3)$ \\
\hline $12+$ & $17(5.8)$ & - & - & - & - & $9(2.9)$ & $10(3.3)$ \\
\hline \multicolumn{8}{|l|}{ Dental visits } \\
\hline Yes & - & - & $109(36.4)$ & - & $145(48.0)$ & $161(53.5)$ & - \\
\hline No & - & - & $190(63.0)$ & - & $156(52.0)$ & $140(46.6)$ & - \\
\hline \multicolumn{8}{|l|}{ Toothbrushing n (\%) } \\
\hline$<2 /$ day & - & - & $33(11.0)$ & - & $67(22.2)$ & $7(2.5)$ & 58 (19.3) \\
\hline$\geq 2 /$ Day & - & - & $268(89.0)$ & - & $234(77.8)$ & $294(97.5)$ & $243(80.7)$ \\
\hline Birth weight (grams), mean (S.D.) & $2968.05(661.7)$ & - & - & - & - & - & - \\
\hline
\end{tabular}


in permanent teeth as measured by the DMFT index and the combination of FT (filled teeth) and DMT (unsound teeth). It can be seen that DMFT increased with age. DMT dropped slightly from age 12 to 18 years due to increased filling during adolescence (Figure 1a). Dental caries experience in deciduous dentition at age 6 affected an average of 3.46 teeth. Participants who did not brush their teeth at least twice a day ranged from $11 \%$ at age 6 to $22 \%$ at age 12 . At 6 years of age, close to two-thirds of the children had never visited a dentist.
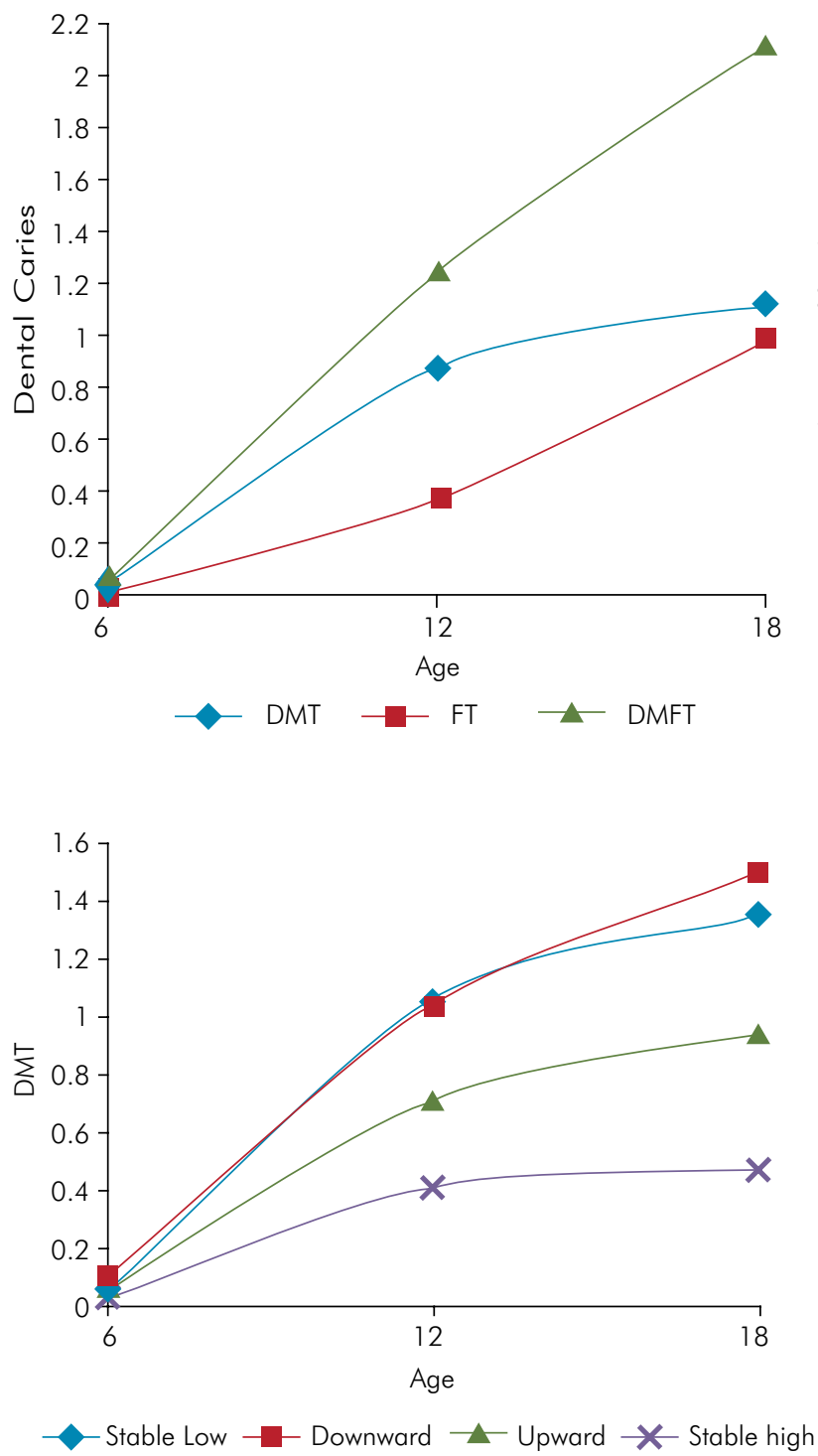

Unadjusted and adjusted dental caries (Incidence Rate Ratio-IRR), calculated by application of the inverse link function from the coefficient $(\beta)$ of the model estimation for income trajectories groups, was estimated for sequentially adjusted models for each of the three outcomes studied. After full adjustment, those from stable low-income trajectory and upward income trajectory groups (the latter with borderline association) presented higher IRR for unsound teeth than those from the stable high-income trajectory group. The stable low-income group presented with lower IRR of filled
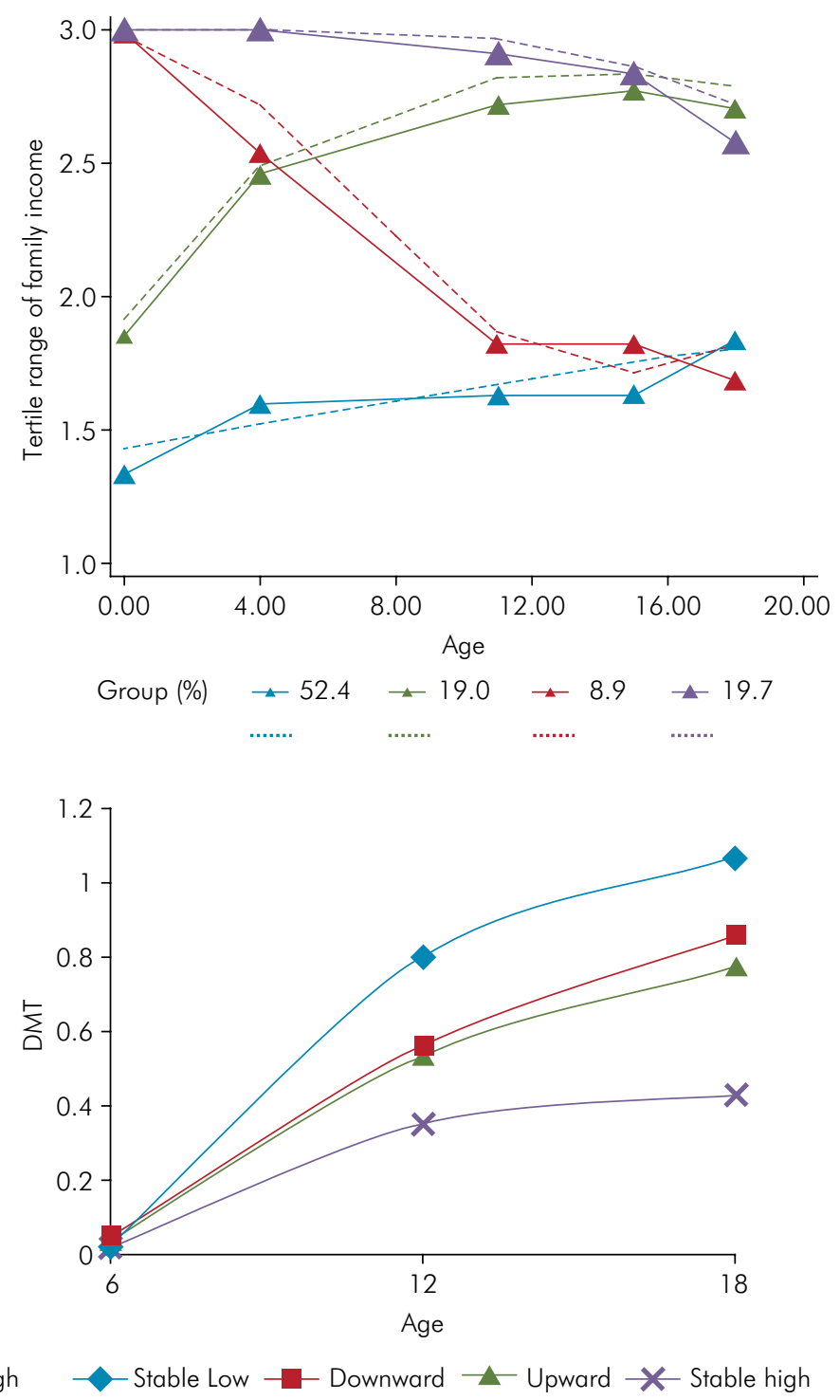

Figure 1. (a) Means or DMT/FT/DMFT. (b) Family income trajectories. (c) Mean DMT calculated from data vs income trajectories. (d) Estimated DMT count with the separate crude model vs income trajectories. 
teeth than those in the stable high-income trajectory group. DMFT was not associated with family income trajectories (Table 2). Generalized Chi-Square can be used as a test of the model form: Are the data overdispersed when the model has a negative binomial distribution?-for example, with model 5 on DMF, $\mathrm{p}=$ $1-$ probchi $(729.77,897)=0.999$. The non-significant $p$ value suggests that the negative binomial model is a good fit for the data. Generalized Chi-Square/DF can be used to indicate how well the model fit the data. A good model fit is indicated when its value is close to one. For our models, the statistics suggested that the model fit the data very well for DM and DMF, but for $\mathrm{F}$, the models were relatively moderate.

Table 3 shows comparisons between two-by-two income trajectory groups for unadjusted (Model 1) and fully adjusted models (Model 5) for the three outcomes studied. After adjustment, the stable low-income group had higher DMT than the stable high-income group; marginal differences were found between the low-income group and the upward-income group, and between the upward-income group and the stable high-income group. The robust difference between the stable low- and stable highincome groups was maintained for FT but in the opposite direction; the stable low-income trajectory group had fewer treated teeth than the stable highincome group. The stable low-income group presented with higher DMFT than the stable high-income group.

Figure 1a displays the observed mean plot of dental caries increase from ages 6 to 18 that suggests caries increase as a quadratic function of age. Dental caries experience increased linearly from the ages of 6 to 18, while FT increased from age 6 but markedly from age 12. The downward income group had the highest DMFT at age 18 followed by the stable low-, upward, and the stable high-income groups, but these were

Table 2. Fixed effects of negative binomial models on the association between dental caries increment and family income trajectories.

\begin{tabular}{|c|c|c|c|c|c|c|}
\hline \multirow{2}{*}{ Income trajectory } & \multicolumn{2}{|c|}{ DMT } & \multicolumn{2}{|c|}{ FT } & \multicolumn{2}{|c|}{ DMFT } \\
\hline & IRR & $95 \% \mathrm{Cl}$ & IRR & $95 \% \mathrm{Cl}$ & IRR & $95 \% \mathrm{Cl}$ \\
\hline \multicolumn{7}{|l|}{ Model 1} \\
\hline Stable low & 2.67 & $1.74-4.11$ & 0.55 & $0.36-0.84$ & 1.30 & $0.94-1.81$ \\
\hline Downward & 2.00 & $1.07-3.72$ & 1.05 & $0.56-1.97$ & 1.37 & $0.84-2.25$ \\
\hline Upward & 1.76 & $1.04-2.98$ & 0.75 & $0.44-1.28$ & 1.13 & $0.75-1.70$ \\
\hline Stable high & 1.00 & & 1.00 & & 1.00 & \\
\hline -2 Res Log Pseudo-likelihood & & 3897.45 & & 4430.50 & & 3596.83 \\
\hline Generalized Chi-Square & & 768.00 & & 511.05 & & 824.03 \\
\hline Gener. Chi-Square / DF & & 0.86 & & 0.57 & & 0.92 \\
\hline \multicolumn{7}{|l|}{ Model 2} \\
\hline Stable low & 2.59 & $1.68-3.99$ & 0.55 & $0.36-0.84$ & 1.26 & $0.91-1.76$ \\
\hline Downward & 1.92 & $1.03-3.59$ & 1.05 & $0.56-1.97$ & 1.32 & $0.81-2.17$ \\
\hline Upward & 1.74 & $1.03-2.96$ & 0.75 & $0.44-1.28$ & 1.12 & $0.74-1.69$ \\
\hline Stable high & 1.00 & & 1.00 & & 1.00 & \\
\hline -2 Res Log Pseudo-likelihood & & 3900.00 & & 4432.87 & & 3601.56 \\
\hline Generalized Chi-Square & & 760.94 & & 508.96 & & 821.70 \\
\hline Gener. Chi-Square / DF & & 0.86 & & 0.57 & & 0.92 \\
\hline \multicolumn{7}{|l|}{ Model 3} \\
\hline Stable low & 2.28 & $1.46-3.56$ & 0.55 & $0.36-0.84$ & 1.17 & $0.83-1.65$ \\
\hline Downward & 1.78 & $0.95-3.34$ & 1.05 & $0.56-1.97$ & 1.26 & $0.76-2.08$ \\
\hline Upward & 1.62 & $0.95-2.76$ & 0.75 & $0.44-1.28$ & 1.07 & $0.70-1.62$ \\
\hline Stable high & 1.00 & & 1.00 & & 1.00 & \\
\hline
\end{tabular}

IRR: incident rate ratio; $\mathrm{Cl}$ : confident interval.

Note: Pseudo-BIC for Model 1 (3943.95, 4495.90, 3641.85) ...; Pseudo-BIC for Model 5 (3913.65, 4524.52, 3609.04); Models built using step forward and the criteria of keeping a variable in the models if it's type III likelihood ratio tests $p$ value less than or equals 0.20 (except Sex); Type III likelihood ratio test in the models ${ }^{*} p<0.5 ;{ }^{* *} p<0.01 ;{ }^{* * *} \mathrm{p}<0.001,+\mathrm{P}<0.10$; All $95 \% \mathrm{Cl}$ derived from Wald Chi-square statistic test; Model 1 (DMT/FT/DMFT), Family income trajectories with Age as time variable, which has been rescaled from 6-12-18 to 1-2-3; Model 2 (DMT/FT/DMFT), Model 1 plus Sex and Birth weight, which was dropped for FT; Model 3 (DMT/FT/DMFT), Model 2 plus Mother's education trajectory but it was dropped for FT; Model 4 (DMT/FT/DMFT), Model 3 plus deciduous tooth caries experience (dmft index) at age 6. Mother's education was dropped from DMFT; Model 5 (DMT/FT/DMFT), Model 4 plus Tooth brushing trajectory and Dental visits at age 6, 12 and 15. 
Table 3. Cross-reference between income trajectories.*

\begin{tabular}{|c|c|c|c|c|c|c|c|}
\hline \multirow{2}{*}{ Variable } & \multirow{2}{*}{ Trajectory group } & \multicolumn{2}{|c|}{ DMT } & \multicolumn{2}{|c|}{ FT } & \multicolumn{2}{|c|}{ DMFT } \\
\hline & & IRR (SE) & $p$-value & IRR (SE) & $\mathrm{p}$-value & IRR (SE) & $\mathrm{p}$-value \\
\hline \multirow{9}{*}{ Model 1} & Stable low (1) & & & & & & \\
\hline & Downward (2) & $1.34(1.30)$ & 0.28 & $0.52(1.34)$ & 0.02 & $0.95(1.25)$ & 0.80 \\
\hline & Upward (3) & $1.52(1.23)$ & 0.04 & $0.73(1.27)$ & 0.18 & $1.15(1.19)$ & 0.41 \\
\hline & Stable high (4) & $2.67(1.25)$ & $<.001$ & $0.55(1.24)$ & 0.01 & $1.30(1.18)$ & 0.12 \\
\hline & Downward (2) & & & & & & \\
\hline & Upward (3) & $1.14(1.36)$ & 0.68 & $1.40(1.39)$ & 0.31 & $1.22(1.29)$ & 0.44 \\
\hline & Stable high (4) & $2.00(1.37)$ & 0.03 & $1.05(1.38)$ & 0.87 & $1.37(1.29)$ & 0.21 \\
\hline & Upward (3) & & & & & & \\
\hline & Stable high (4) & $1.76(1.31)$ & 0.04 & $0.75(1.31)$ & 0.29 & $1.13(1.23)$ & 0.57 \\
\hline \multirow{8}{*}{ Model 5} & Stable low (1) & & & & & & \\
\hline & Downward (2) & $1.38(1.28)$ & 0.19 & $0.64(1.34)$ & 0.13 & $0.05(0.20)$ & 0.82 \\
\hline & Upward (3) & $1.44(1.22)$ & 0.07 & $0.88(1.28)$ & 0.61 & $0.18(0.17)$ & 0.28 \\
\hline & Stable high (4) & $2.36(1.25)$ & $<0.01$ & $0.64(1.26)$ & 0.06 & $0.21(0.17)$ & 0.20 \\
\hline & Downward (2) & & & & & & \\
\hline & Upward (3) & $1.04(1.33)$ & 0.88 & $1.38(1.40)$ & 0.34 & $0.13(0.24)$ & 0.57 \\
\hline & Stable high (4) & $1.71(1.35)$ & 0.08 & 1.01 (1.38) & 0.98 & $0.17(0.23)$ & 0.48 \\
\hline & Stable high (4) & $1.64(1.30)$ & 0.06 & $0.73(1.31)$ & 0.25 & $0.03(0.20)$ & 0.87 \\
\hline
\end{tabular}

*IRR of dummy variable in the first column refers to the next column $(=1)$. For example, the incident rate of DMT for Stable low is 2.67 higher than Stable high, and for FT, Stable low is just over half (0.55) the value of Stable high, or the FT rate of Stable high is $1.82(=1 / 0.55)$ higher than that of the Stable low group.

not statistically different. Figures 1a and 1c show that the observed mean plot of DMT increased from ages 6 to 18 versus family income categories. The estimate model showed that, during the life-course, stable low- and downward income groups had the highest DMT values followed by upward and stable high-income groups (Figure 1d).

\section{Discussion}

Family income trajectories in the life-course affected increases in treated (FT) and untreated dental caries (DMT) from childhood to adulthood. Those participants placed in the stable low- and upward income groups presented with higher levels of unsound teeth than did those in the stable high-income group; the upward income group presented with slightly fewer unsound teeth than those in the stable low-income group. The stable low-income group had a lower level of filled teeth than the stable high-income group in the lifecourse. Experience of dental caries, as expressed by DMFT, was not affected by income trajectories. The inconsistency of DMT between Figure 1c and Figure $1 \mathrm{~d}$ on the downward trajectory is evidence that the mean value is not a reliable measure of caries data, due to its skewness, especially with a small sample.

Family income gradients were identified mainly across unsound (DMT) and 'treated' (FT) components of the dental caries index - DMFT - suggesting that income inequalities in dental caries management is more apparent than in disease experience, corroborating the results of a previous study undertaken in Australia. ${ }^{21}$

It is well-known that persistent relative poverty is associated with disease. However, our findings also suggest that experience of at least one episode of relative poverty, characterized by mobile income groups - downward and upward - is sufficient to increase the levels of untreated dental caries from childhood to adolescence, corroborating findings from the 1982 Pelotas cohort study. ${ }^{7}$ Paradoxically, this occurred in the context of a universal public health system suggesting the existence of inequity in the pattern of dental attendance according to different income trajectories. In fact, despite the existence of a universal and free-of-charge National Health System, almost half of Brazilians pay outofpocket for dental services. ${ }^{22}$ 
To the best of our knowledge, this is the only study, along with The Dunedin Study, ${ }^{5}$ that simultaneously investigated income and dental caries trajectories from childhood to young adulthood. However, our study modeled both income and increases in dental caries longitudinally. The main strengths of this study are the prospective design of a representative sample of newborns and the longitudinal analyses of three dental caries assessments and five income assessments by statistical methods that account for loss of information. High reliabilities of clinical data and adjustment for potential mediators and confounders are other methodological strengths of this study. Ideally, the best way to account for family size is to calculate equivalized income, which is a measure of income that gives weight to children under 15 years of age and adults over 60 years of age. Since family unit size information was not available at birth and age 4 years, we could not calculate equivalized income. However, when analyzing family size units at ages 11, 15 , and 18 years, we did not find significant differences.

Two approaches are commonly used for modeling repeated measures in longitudinal data: randomeffects models (or mixed models), which use maximum likelihood estimation; and population average models, which typically use a generalized estimation equation (GEE) approach with quasi-likelihood estimation. In many cohort studies, a dependent variable, Y, measured repeatedly at the lower (individual) level, is assumed to be influenced by explanatory variable $X$, also measured at the lower level, and by explanatory variable Z, measured at the higher level. If possible, $X$ should be measured during the study, not necessarily concurrently with the measurements of Y. Timevarying explanatory variable(s) can be successfully handled in both mixed models and GEE models if the time-varying independent variable(s) are simultaneously measured (or transformed to) with the dependent variable in the same time scale. In this study, we used GLMM to analyze a birth cohort with dental caries data as the dependent variable measured at three waves when the study participants were 6, 12, and 18 years of age. Potential explanatory variables were collected concurrently when dental caries was examined and at different time points. Family income, mother's education, and toothbrushing frequency were integrated with higher (group) levels through trajectory modeling. Since the distribution of dental caries indices is notoriously positively skewed, it was not appropriate to use traditional linear models. Poisson and negative binomial models have been used for describing caries count distribution. However, Poisson regression modeling is not recommended with skewed distribution and over-dispersion. Under these circumstances, negative binomial regression modeling is recommended. ${ }^{23}$

Since the coverage of dental care was relatively low, as measured by the proportion of F/DMFT, the dentist's decision for treatment, which in turn may increase the $F$ component and affect dental caries indices, ${ }^{24}$ will likely have little impact on our findings. Dental attendance is relatively low despite the existence of a universal health system in Brazil that includes dental care. However, other factors are more important for the prevention of dental caries, such as the use of fluoride toothpaste and water fluoridation. The city implemented water fluoridation in 1962, covering almost the entire urban population; fluoride toothpaste is almost universal in Brazil, since the government provides it free of charge to those who cannot afford it. Brazil has one of the highest rates of per capita consumption of fluoride toothpaste in the world. ${ }^{25}$

The main limitation of this study is its relatively small sample size, which may have precluded the assessment of some actual associations, particularly those identified with a statistically borderline or marginal significance. The unsound component of 'service usage' ('M') and the lack of 'service usage' ('D') were treated jointly as 'negative outcomes'. This is an analogy to the sound/ unsound teeth definition as used previously.

As identified in the Dunedin study, ${ }^{2}$ caries appears to occur at a relatively constant rate throughout life. However, the pattern of untreated dental caries varies across income trajectory groups. The slope of the DMT curve markedly increased in stable and downward groups, particularly between 6 and 12 years of age, while for upward and stable high-income groups there was an increase in DMT from 6 to 12 years of age, after which a plateau can be identified.

West ${ }^{26}$ and West et al. ${ }^{27}$ examined empirical data on general morbidity and mortality in the UK and postulated that there is a relative equality of health in childhood and adolescence. Our findings challenge 
this notion in youth. We found significant differences in the levels of unsound teeth from childhood to young adulthood across different income trajectories. These findings are in line with those found at the national level, which identified that the proportion of individuals who had never visited a dentist in each income stratum was more unequal among younger age groups. ${ }^{22}$

We applied a predictive model to test whether income trajectories affect dental caries increases in the life-course. The next step is to try to understand the mechanism beyond this association, the potential mediation role of behavior and dental visiting, by the application of an explanatory model. ${ }^{28}$

\section{Conclusion}

It is possible to conclude that differences across income trajectory groups were found in the management of dental caries-treated and untreatedrather than in the experience of disease.

\section{Acknowledgements}

This article is based on data from the Pelotas Birth Cohort, 1993, study, conducted by the Postgraduate Program in Epidemiology at the Universidade Federal de Pelotas. The 1993 birth cohort study is currently supported by the Wellcome Trust through the program entitled Major Awards for Latin America on Health Consequences of Population Change. The European Union, National Support Program for Centers of Excellence (PRONEX), the Brazilian National Research Council (CNPq), and the Brazilian Ministry of Health supported previous phases of the study. Oral health assessment at age 12 was sponsored by the Brazilian National Council for Scientific and Technological Development - CNPq (grant No 403362/2004-0).

\section{References}

1. Thomson WM, Poulton R, Milne BJ, Caspi A, Broughton $J R$, Ayers KM. Socioeconomic inequalities in oral health in childhood and adulthood in a birth cohort. Community Dent Oral Epidemiol. 2004 Oct;32(5):345-53 https://doi.org/10.1111/j.1600-0528.2004.00173.x

2. Broadbent JM, Thomson WM, Poulton R. Trajectory patterns of dental caries experience in the permanent dentition to the fourth decade of life. J Dent Res. 2008 Jan;87(1):69-72. https://doi.org/10.1177/154405910808700112

3. Marcenes W, Kassebaum NJ, Bernabé E, Flaxman A, Naghavi M, Lopez A et al. Global burden of oral conditions in 1990 2010: a systematic analysis. J Dent Res. 2013 Jul;92(7):592-7. https://doi.org/10.1177/0022034513490168

4. Sgan-Cohen HD, Evans RW, Whelton H, Villena RS, MacDougall M, Williams DM et al.; IADR-GOHIRA Steering and Task Groups. IADR Global Oral Health Inequalities Research Agenda (IADR-GOHIRA(R)): a call to action. J Dent Res. 2013 Mar;92(3):209-11. https://doi.org/10.1177/0022034512475214

5. Poulton R, Caspi A, Milne BJ, Thomson WM, Taylor A, Sears MR et al. Association between children's experience of socioeconomic disadvantage and adult health: a lifecourse study. Lancet. 2002 Nov;360(9346):1640-5. https://doi.org/10.1016/S0140-6736(02)11602-3

6. Peres MA, Peres KG, Barros AJ, Victora CG. The relation between family socioeconomic trajectories from childhood to adolescence and dental caries and associated oral behaviours. J Epidemiol Community Health. 2007 Feb;61(2):141-5. https://doi.org/10.1136/jech.2005.044818

7. Peres MA, Peres KG, Thomson WM, Broadbent JM, Gigante DP, Horta BL. The influence of family income trajectories from birth to adulthood on adult oral health: findings from the 1982 Pelotas birth cohort. Am J Public Health. 2011 Apr;101(4):730-6. https://doi.org/10.2105/AJPH.2009.184044

8. Johnson-Lawrence V, Kaplan G, Galea S. Socioeconomic mobility in adulthood and cardiovascular disease mortality. Ann Epidemiol. 2013 Apr;23(4):167-71. https://doi.org/10.1016/i.annepidem.2013.02.004

9. Victora CG, Hallal PC, Araújo CL, Menezes AM, Wells JC, Barros FC. Cohort profile: the 1993 Pelotas (Brazil) birth cohort study. Int J Epidemiol. 2008 Aug;37(4):704-9. https://doi.org/10.1093/ije/dym177

10. Peres MA, Barros AJ, Peres KG, Araújo CL, Menezes AM, Hallal PC et al. Oral health follow-up studies in the 1993 Pelotas (Brazil) birth cohort study: methodology and principal results. Cad Saude Publica. 2010 Oct;26(10):1990-9. https://doi.org/10.1590/S0102-311X2010001000016

11. Gonçalves $H$, Assunção MC, Wehrmeister FC, Oliveira IO, Barros FC, Victora CG et al. Cohort profile update: The 1993 Pelotas (Brazil) birth cohort follow-up visits in adolescence. Int J Epidemiol. 2014 Aug;43(4):1082-8. https://doi.org/10.1093/ije/dyu077 
12. Peres MA, Barros AJ, Peres KG, Araújo CL, Menezes AM. Life course dental caries determinants and predictors in children aged 12 years: a population-based birth cohort. Community Dent Oral Epidemiol. 2009 Apr;37(2):123-33. https://doi.org/10.1111/j.1600-0528.2009.00460.xPMID:19250294

13. Peres MA, Latorre MRO, Sheiham A, Peres KG, Barros FC, Hernandez PG et al. Social and biological early life influences on severity of dental caries in children aged 6 years. Community Dent Oral Epidemiol. 2005 Feb;33(1):53-63. https://doi.org/10.1111/j.1600-0528.2004.00197.x

14. Peres KG, Latorre MRO, Sheiham A, Peres MA, Victora CG, Barros $F C$. Social and biological early life influences on the prevalence of open bite in Brazilian 6-year-olds. Int J Paediatr Dent. 2007 Jan;17(1):41-9. https://doi.org/10.1111/j.1365-263X.2006.00793.x

15. World Health Organization. Oral health surveys: basic methods. 4th ed. Geneva: World Health Organization; 1997.

16. Peres MA, Traebert J, Marcenes W. [Calibration of examiners for dental caries epidemiologic studies]. Cad Saude Publica. 2001 Jan-Feb;17(1):153-9. Portuguese. https://doi.org/10.1590/S0102-311X2001000100016

17. Jones BL, Nagin DS, Roeder K. A SAS procedure based on mixture models for estimating developmental trajectories. Sociol Methods Res. 2001;29(3):374-93. https://doi.org/10.1177/0049124101029003005

18. Szklo M, Javier-Nieto F. Epidemiology: beyond the basics. 2nd ed. Sudbury: Jones and Bartlett; 2007.

19. Jones BL, Nagin DS. Advances in group-based trajectory modeling and a SAS procedure for estimating them. Sociol Methods Res. 2007;35:542-71.

20. Gurland J. Some applications of the negative binomial and other contagious distribution. Am J Public Health. 1959;49(10):138899. https://doi.org/10.2105/AJPH.49.10.1388
21. Mejia G, Jamieson LM, Ha D, Spencer AJ. Greater inequalities in dental treatment than in disease experience. J Dent Res. 2014 Oct;93(10):966-71. https://doi.org/10.1177/0022034514545516

22. Peres KG, Peres MA, Boing AF, Bertoldi AD, Bastos $\mathrm{JL}$, Barros AJ. Reduction of social inequalities in utilization of dental care in Brazil from 1998 to 2008. Rev Saude Publica. 2012 Apr;46(2):250-8. https://doi.org/10.1590/S0034-89102012000200007

23. Preisser JS, Stamm JW, Long DL, Kincade ME. Review and recommendations for zero-inflated count regression modeling of dental caries indices in epidemiological studies. Caries Res. 2012;46(4):413-23. https://doi.org/10.1159/000338992

24. Marcenes W, Freysleben GR, Peres MA. Contribution of changing diagnostic criteria toward reduction of caries between 1971 and 1997 in children attending the same school in Florianopolis, Brazil. Community Dent Oral Epidemiol. 2001 Dec;29(6):449-55. https://doi.org/10.1034/j.1600-0528.2001.290606.x

25. Cury JA, Tenuta LM, Ribeiro CC, Paes Leme AF. The importance of fluoride dentifrices to the current dental caries prevalence in Brazil. Braz Dent J. 2004;15(3):167-74. https://doi.org/10.1590/S0103-64402004000300001

26. West $P$. Health inequalities in the early years: is there equalisation in youth? Soc Sci Med. 1997 Mar;44(6):833-58. https://doi.org/10.1016/S0277-9536(96)00188-8

27. West $\mathrm{P}$, Sweeting $\mathrm{H}$. Evidence on equalisation in health in youth from the West of Scotland. Soc Sci Med. 2004 Jul;59(1):13-27. https://doi.org/10.1016/j.socscimed.2003.12.004

28. Shmueli G. To explain or to predict? Stat Sci. 2010;25(3):289310. https://doi.org/10.1214/10-STS330 